\title{
SOST polymorphisms and response to alendronate treatment in postmenopausal Chinese women with osteoporosis
}

\begin{abstract}
Aim: To investigate the association between SOST gene polymorphisms and response to alendronate treatment. Materials \& methods: 639 Chinese postmenopausal women with osteoporosis or osteopenia received alendronate treatment. Polymorphisms of SOST were analyzed. Bone mineral density (BMD), serum ALP and $\beta$-CTX levels were measured. The correlation of SOST polymorphisms with changes of BMD and bone biomarkers after treatment was analyzed. Results: rs1234612 and rs851054 polymorphisms were correlated to baseline lumbar spine BMD ( $<0.05)$. After 12 months of treatment rs1234612 and rs865429 polymorphisms were correlated to BMD changes at the lumbar spine $(p<0.05)$ or femoral neck $(p<0.05)$, respectively. Conclusion: The polymorphisms of SOST are genetic factors affecting bone health and response to alendronate in Chinese postmenopausal women.
\end{abstract}

Keywords: osteoporosis $\bullet$ polymorphisms $\bullet$ response to alendronate $\bullet$ SOST gene

\section{Background}

Postmenopausal osteoporosis is a systemic skeletal disorder characterized by progressive bone loss, which results in deteriorated bone microstructure, compromised bone strength and increased risk of fracture [1]. A genetic contribution to osteoporosis is well established, of which linkage and genome-wide association studies have identified several loci which influence bone mineral density (BMD) variation or fracture risk [2-5].

As we know, bisphosphonates (BPs) are appropriate first-line therapy for osteoporosis, which is characterized by differing clinical responses. In terms of BMD, about $26-53 \%$ of patients do not respond to BPs therapy [6]. Identification of the factors that predict responsiveness to BPs may contribute to the avoidance of unnecessary BPs treatment and result in a significant decrease in medical costs. Sequence variants in the human genome are now considered as important causes of differences in drug responses [7]. To date, there are few, small sampled studies on pharmacogenetics of osteoporosis, in which polymorphisms of $V D R$ [8], ERß [9], LRP5 [10], FDPS [11], $D K K 1$ [12] have been investigated with regard to response to antiosteoporotic agents, such as hormone-replacement therapy, raloxifene or BPs. However, the genes that may determine bone response to BPs have not yet been precisely defined.

The effects of antiosteoporotic treatment are determined by status of the remodeling process, which involves both bone resorption by osteoclasts and bone formation by osteoblasts. Stimulation of bone formation is necessary to achieve improvements in bone mass, architecture and strength. The wingless integration (Wnt) signaling pathway has been well known as a key regulatory component of bone formation [13]. Sclerostin, an osteocyte-derived inhibitor of osteoblast activity, antagonizes Wnt signaling in both osteocytes and osteoblasts by binding to the lipoprotein receptor-related protein (LRP5/G) coreceptor $[14,15]$. Sclerostin is encoded by SOST gene, which locates on chromosome 17q12q21 [13]. Loss-of-function mutations of SOST lead to rare genetic skeletal disorders such as sclerosteosis and Van Buchem disease, asso-
Pei-ran Zhou', Xiao-jie Xu', Zhen-lin Zhang', Er-yuan $\mathrm{Liao}^{3}$, De-cai Chen ${ }^{4}$, Jian Liu ${ }^{5}$, Wen $\mathrm{Wu}^{6}$, Yan Jiang', Ou Wang', Wei-bo Xia', Xiao-ping Xing ${ }^{1}$, Ling $\mathrm{Xu}^{7}$ \& Mei Li ${ }^{\star}, 1$

'Department of Endocrinology, Key Laboratory of Endocrinology of Ministry of Health, Peking Union Medical College Hospital, Chinese Academy of Medical

Sciences, Beijing, China

${ }^{2}$ Metabolic Bone Disease \& Genetics Research Unit, Department of

Osteoporosis \& Bone Diseases, Shanghai Sixth People's Hospital Affiliated with

Shanghai Jiao Tong University, Shanghai, China

${ }^{3}$ Institute of Endocrinology \&

Metabolism, Second Xiangya Hospital of Central South University, Changsha,

Hunan, China

${ }^{4}$ Department of Endocrinology, Osteoporosis Education Center, West China Hospital, Sichuan University, Chengdu, Sichuan, China

${ }^{5}$ Department of Orthopedics, Xijing Hospital, The Fourth Liberation Army University, Xi'an, Shaanxi, China ${ }^{6}$ Department of Endocrinology, Guangdong General Hospital, Guangzhou, Guangdong, China 7Department of Obstetrics \& Gynecology, Peking Union Medical College Hospital, Peking Union Medical College, Chinese Academy of Medical Sciences, Beijing, China

*Author for correspondence:

Tel.: +86106915 5088

Fax: +86106915 5088

limeilzh@sina.com

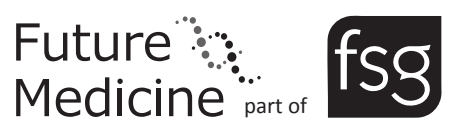


ciating with a high BMD phenotype and low risk of fractures [16]. Antisclerostin antibodies were associated with increased BMD and bone formation $[17,18]$. As one of the most crucial regulators of Wnt pathway and a new treatment target of osteoporosis, it is valuable to assess the possible relevance of SOST variance and bone response to antiosteoporotic agents. The polymorphisms of SOST gene were demonstrated to be strongly associated with BMD of postmenopausal Chinese women [19], and were correlated to body composition and BMD in young and elderly Men [20]. However, correlation between genetic variance of SOST and the response to BPs treatment are still scanty.

As we know, alendronate is a nitrogen-containing bisphosphonate, and the most widely used in the treatment of postmenopausal osteoporosis, with a proven efficacy in reduction of bone resorption and fragility fractures [21]. However, little is known about the possible influence of genetic factors on the response to alendronate. Therefore, we investigated the relationship between polymorphisms of SOST gene and the effects of alendronate on BMD and bone turnover biomarkers in postmenopausal Chinese women.

\section{Materials \& methods \\ Study participants}

This was an open-label, multicenter, prospective study. During 2008 to 2011 more than 2000 postmenopausal women were screened from seven clinical centers in China (Beijing, Changsha, Shanghai, Chengdu, Xi'an, Guangzhou and Haerbin). A total of 639 postmenopausal women between the age of 41 and 75 years diagnosed as osteoporosis or osteopenia were enrolled. As previously reported $[10,11]$, the inclusion criteria were as follows: years since menopause (YSM) more than 1 year and age at menopause older than 40 years; BMI between 18 and $35 \mathrm{~kg} / \mathrm{m}^{2}$; T score of BMD at lumbar spine or femoral neck less than -1; at least three lumbar vertebrae were measurable. Exclusion criteria were: intolerance to BPs; with serious chronic diseases of liver, kidney, esophagus or stomach; treatment with BPs or teriparatide within previous 12 months, and treatment with estrogen, selective estrogen receptor modulators or calcitonin within previous 6 months; taken other drugs affecting bone metabolism within previous 3 months; with other metabolic bone diseases, connective tissue disease or malignant tumor. The study was approved by the Ethic Committee of Peking Union Medical College Hospital and the local Ethic Committee of each center. All participants were voluntary, and signed the informed consent.

Participants with osteoporosis or osteopenia randomly received treatment of standard dose $(70 \mathrm{mg}$ weekly) or low dose (70 mg every 2 weeks) of alen- dronate (Ouyi Pharmaceutical Co., Ltd, Shijiazhuang, China) for 12 months. All women were supplemented with $600 \mathrm{mg}$ elemental calcium and 125 IU vitamin D3 (Caltrate with vitamin D 600 tablets, Pfizer Inc., NJ, USA) daily. Compliance to alendronate was evaluated by the number of empty medicine bottles and the remaining tablets at each follow-up.

\section{SNP selection \& genotyping}

Single nuclear polymorphisms (SNPs) information of SOST gene was obtained from Entrez Gene database (www.ncbi.nlm.nih.gov/gene) and International HapMap Project Data Release 27 (February 2009) (http://hapmap.ncbi.nlm.nih.gov/cgi-perl/gbrowse/ hapmap27_B36). SNPs were selected via Haploview 4.0 [22] from a region on chromosome 17 between base pairs 39181625 and 39196682 according to the following criteria: minor allele frequency higher than $10 \%$ in Chinese Han population; classified as tag SNP and with pairwise linkage disequilibrium exceeding the threshold of $0.8\left(r^{2}>0.8\right)$; covering both upstream and downstream untranslated regions of SOST gene and loci reported to be associated with BMD [23,24]. Finally, six tag SNPs and one positive SNP of SOST were selected: rs1234612, rs851054, rs865429, rs851062, rs1513670, rs9902563 and rs1877632 (Supplementary Table 1). The Haploview linkage disequilibrium plots for all SNPs in the SOST were shown in Figure 1.

Genomic DNA was extracted from peripheral leukocytes with a QIAamp blood kit (QIAGEN Inc., CA, USA). Each participant was genotyped for all SNPs of SOST by TaqMan allelic discrimination assay (Applied Biosystems, CA, USA). The whole reacting volume was $5 \mu \mathrm{l}$, including $2 \mu \mathrm{l}$ DNA sample, $2.5 \mu \mathrm{l}$ TaqMan Universal PCR Master Mix, $0.125 \mu \mathrm{l}$ TaqMan probe and $0.375 \mu \mathrm{ldd} \mathrm{H}_{2} \mathrm{O}$. Reactions were performed on a Real-Time PCR system of ABI Prism 7900 (Applied Biosystems, CA, USA) in a 384-well reaction plate under standard conditions. 50 PCR products were directly sequenced to confirm the accuracy of TaqMan allelic discrimination assay.

\section{Assessment of response to alendronate treatment}

The response to alendronate treatment was assessed by percentage changes of BMD and serum bone turnover biomarkers levels from baseline to 12 months of treatment. BMD at lumbar spine $\mathrm{L}_{2}-\mathrm{L}_{4}(\mathrm{LS})$, femoral neck (FN) and total hip (TH) was measured at 0 and 12 months of alendronate treatment by dual-energy x-ray absorptiometry (DXA, Hologicor Lunar). The coefficient variations $(\mathrm{CV})$ of seven centers were $0.75-1.7 \%$ for LS, $0.56-1.0 \%$ for $\mathrm{FN}$, and $0.39-0.95 \%$ for $\mathrm{TH}$. BMD value acquired on different scanners was stan- 
dardized according to the formula: for lumbar spine BMD value, Lunar BMD $\left(\mathrm{g} / \mathrm{cm}^{2}\right)=1.074 \times$ Hologic $+0.054(\mathrm{r}=0.987, \mathrm{p}<0.05$, standard error of the estimate $\left.=0.030 \mathrm{~g} / \mathrm{cm}^{2}\right)$; for hip BMD value: hip BMD $\left(\mathrm{g} / \mathrm{cm}^{2}\right)=1.013 \times$ Hologic $+0.142(\mathrm{r}=0.920, \mathrm{p}<$ 0.05 , standard error of the estimate $\left.=0.051 \mathrm{~g} / \mathrm{cm}^{2}\right)$ [25] Since compression fractures of vertebrae could lead to pseudoincrease in BMD, BMD at lumbar spine were analyzed only on vertebrae without fractures. Lateral radiographs of thoracolumbar spine (from the 4th thoracic vertebra to the 5 th lumbar vertebra) were taken before and after 12 months of treatment to identify vertebral fractures. Vertebral fractures were diagnosed by two experienced radiologists independently, using Genant's semiquantitative method. The radiologists were blinded to the participants' group assignments. According to the criteria of $\mathrm{WHO}$, osteoporosis is defined as BMD T score less than -2.5 or with fragile fracture history, and osteopenia is considered as BMD $\mathrm{T}$ score between -1.0 and -2.5 .

Fasting blood samples were collected from participants at 0,3 and 12 months of treatment. Serum levels of cross linked C-telopeptide of type I collagen ( $\beta$-CTX, as bone resorption marker) and 25 hydroxy-vitamin D (25OHD) were detected by a fully automatic electrochemiluminescence system (E170, Roche Diagnostics, Switzerland) at Peking Union Medical College Hospital simultaneously. The minimal detection limit of $\beta$-CTX and 25OHD was $0.01 \mathrm{ng} / \mathrm{ml}$ and $4 \mathrm{ng} / \mathrm{ml}$, respectively. The intra-assay and interassay CV were 2.0 and $3.1 \%$ for $\beta$-CTX, 5.7 and $6.1 \%$ for $25 \mathrm{OHD}$, respectively. Serum levels of calcium, phosphorus, alkaline phosphatase (ALP, as bone formation marker), alanine aminotransferase and creatinine were detected by automatic analyzer in local centers.

\section{Statistics analysis}

Normally distributed data were expressed as mean \pm standard deviation and abnormally distributed data were expressed as median and quartiles. The $\chi^{2}$ test was used to check the deviation from Hardy-Weinberg equilibrium. Comparisons between the two dosage groups were performed using the student's t-test. For analysis of participants' response to treatment, percentage changes in BMD and bone turnover markers between baseline and follow-up were used, which were calculated by the following formula (e.g., BMD): percentage change in $\mathrm{BMD}=(\mathrm{BMD}$ after treatment - BMD at baseline) / BMD at baseline $\times 100 \%$. Genotype-specific differences in changes of BMD and bone turnover biomarkers were analyzed by general linear model-analysis of covariance (GLM-ANCOVA) with adjustment for confounding factors such as age, YSM,

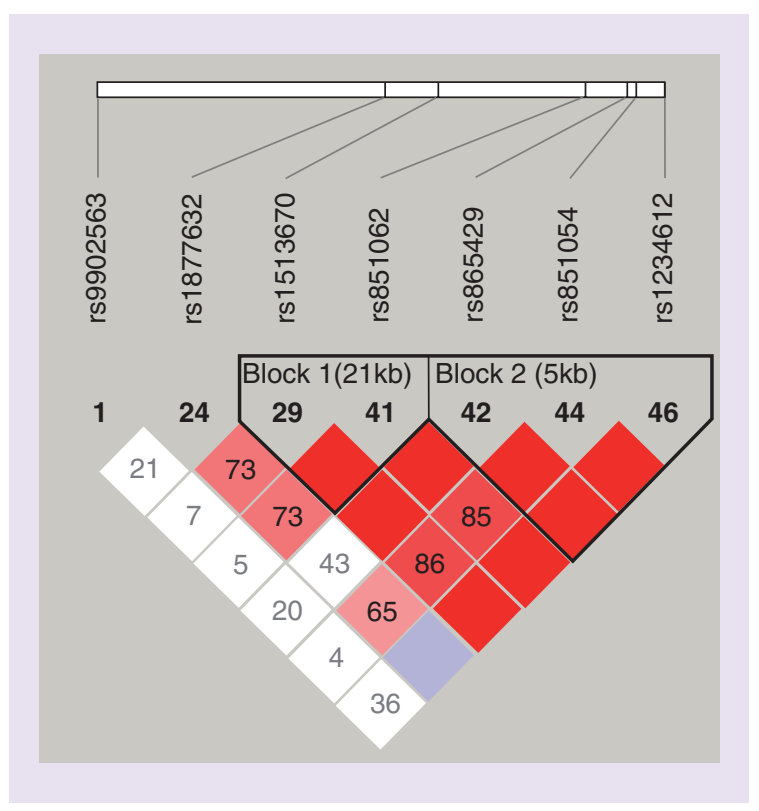

Figure 1. Haploview linkage disequilibrium plots with $R^{2}$ values of the SOST gene using the seven selected SNPs inSOST.

BMI, dosage of medication, location of participants, 25OHD levels and baseline BMD. Comparisons between the genotypes were performed using GLMANCOVA or Kruskal-Wallis test as appropriate. Multiple linear regression was used to analyze the associations between BMD and the genotypes, age, YSM, $\mathrm{BMI}$, the dosage of alendronate, the research center, baseline 25OHD level.

Statistical analysis was performed using the statistical package SPSS 20.0 (SPSS Inc., Chicago, IL, USA). A p-value $<0.05$ was considered to be significant for all analyses.

\section{Results}

Baseline characteristics of participants \& effect of alendronate treatment

A total of 639 women with postmenopausal osteoporosis or osteopenia were enrolled in this study (Figure 2). The baseline demographic characteristics were shown in Table 1. There were no significant differences in all variables between the two groups (Table 1). Previous fractures occurred in 33 and 36 women in the low-dose and standard-dose groups, respectively $(\mathrm{p}=0.271)$.

$540(84.5 \%)$ women completed the 12 months of alendronate treatment. 99 (15.5\%) women dropped out due to clinical adverse effects, protocol deviation, withdrawal of consent and other social reasons. Among them, 60 women dropped out because of gastrointestinal symptoms such as heartburn and stomach pain. These symptoms were mild and transient, which relieved after stopping the treatment. No significant difference in withdrawal rate was found among dif- 
ferent genotypes of SOST. The average adherence to alendronate was $86.6 \%$ assessed by the number of the remaining tablets at each follow-up. After 12 months of treatment, BMD at all sites was significantly increased and serum levels of bone turnover markers were obviously decreased in the two groups (all p < 0.01 ; Table 1). No differences in percentage changes of BMD at LS, FN and TH were found between the lowdose group (5.6, 3.9 and $3.3 \%$, respectively) and the standard-dose group (5.1, 2.9 and 3.8\%, respectively; all $\mathrm{p}>0.05$; Table 1).

Because no difference existed in baseline BMD and the percentage changes after 12 months of treatment between low and standard dosage groups [26], the effects of two dosages of alendronate were similar. In order to avoid the influence of small sample size, we put the two groups together as one sample to analyze the influence of SOST gene polymorphisms on BMD and the response to alendronate. However, we considered effects of alendronate dosage during statistical analysis.

\section{Polymorphisms of SOST \& bone phenotypes} A total of $545(85.3 \%)$ DNA samples were suitable for genetic analysis. The successfully genotyping rate of all seven SOST polymorphisms exceeded 96\% (Supplementary Table 1). All genotype frequencies of SOST did not deviate from Hardy-Weinberg equilibrium ( $p>0.01$ ), and minor allele frequencies exhibited in our cohort were calculated in Supplementary Table 1.

At baseline, LS BMD was associated with rs1234612 genotypes of SOST ( $\mathrm{p}=0.044$, Table 2). Participants with homozygous common alleles had higher LS BMD than those with heterozygous genotypes (TT/TC, $\mathrm{p}=$ 0.025). Participants with homozygous minor alleles presented trend of lower LS BMD than heterozygous genotypes $(\mathrm{CC} / \mathrm{TC}, \mathrm{p}=0.054)$. Genotypes at rs851054 of SOST were associated with baseline serum ALP levels and LS BMD ( $\mathrm{p}=0.003$ and 0.020 , respectively, Table 2). Participants with homozygous minor alleles showed higher serum ALP levels and LS BMD than those with other genotypes (ALP: AA/GA, $p=0.002$, AA/GG, $\mathrm{p}=0.001$; LS BMD: AA/GA, $\mathrm{p}=0.005$, AA/

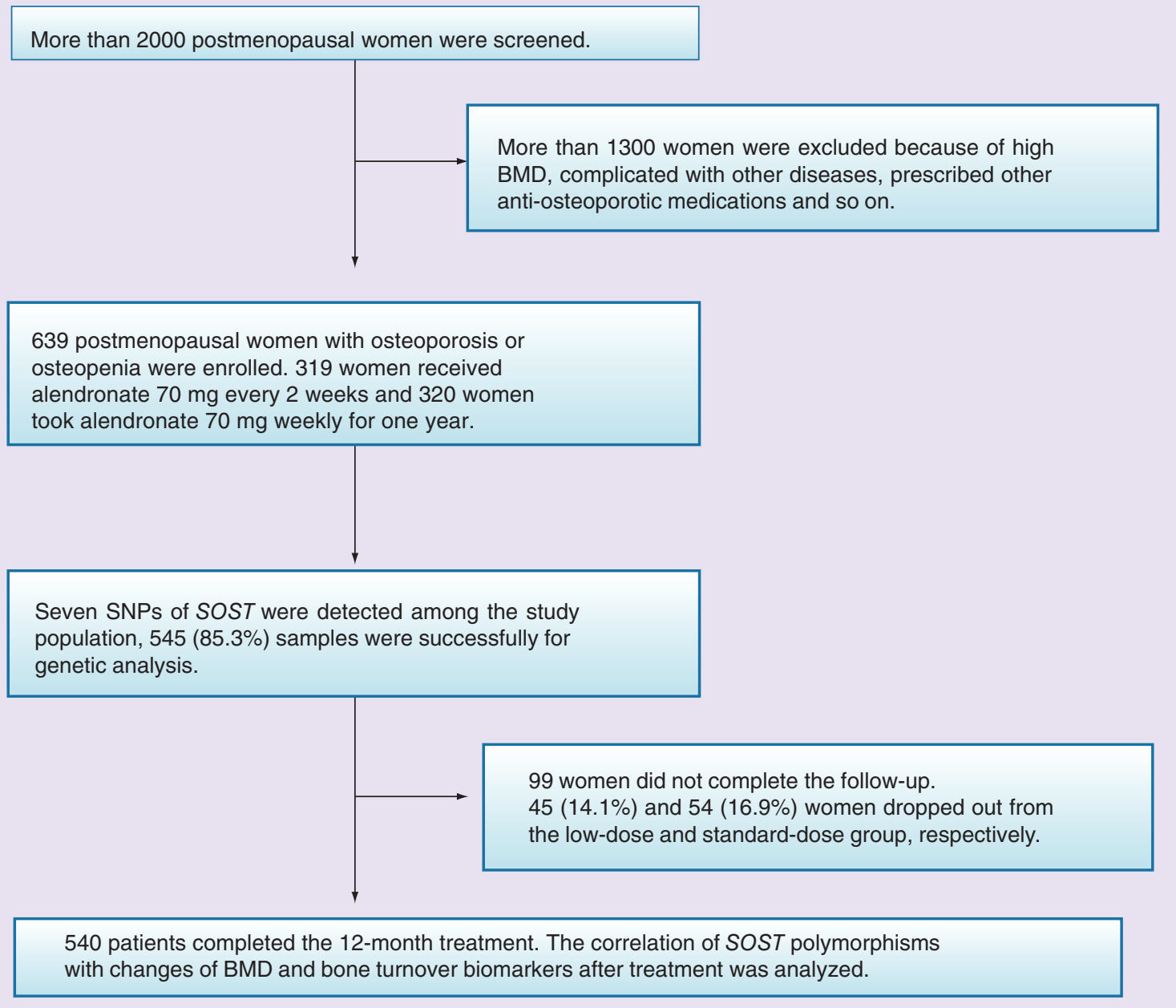

Figure 2. Flow chart of this study. 
Table 1. Comparisons between the two dosage groups before and after alendronate treatment.

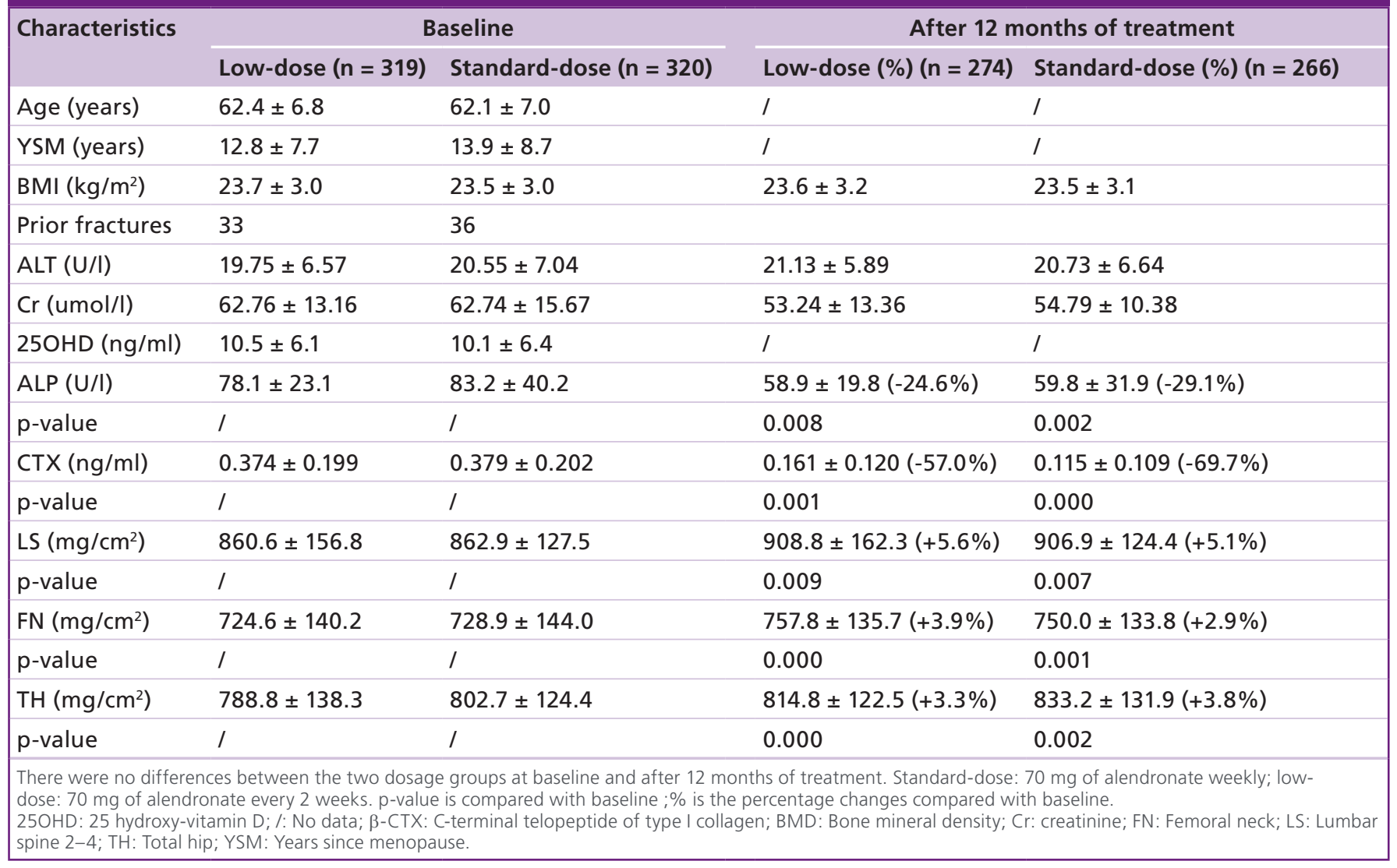

$\mathrm{GG}, \mathrm{p}=0.036)$. Genotypes at the other SNPs loci were not associated either with BMD, or with bone turnover biomarkers at baseline (Supplementary Table 2). Multiple linear regression indicated that baseline lumbar spine BMD was correlated to rs1234612 polymorphisms of SOST ( $\left.\mathrm{p}=0.032, \mathrm{R}^{2}=0.012\right)$, and correlated to rs851054 polymorphisms of SOST ( $\mathrm{p}=0.007$, $\left.\mathrm{R}^{2}=0.017\right)$.

\section{Polymorphisms of SOST \& bone response to alendronate}

After 3 months of treatment, genotypes at only rs1513670 locus were associated with percentage changes in serum ALP levels $(p=0.005)$. However, this association did not remain to 12 months of treatment. Genotypes at the other SNPs loci were uncorrelated to percentage change of ALP or $\beta$-CTX after alendronate treatment.

After 12 months of alendronate treatment, genotypes at rs1234612 locus were associated with percentage change of LS BMD ( $\mathrm{p}=0.015$; Figure 3 \& Table 3 ). Participants with homozygous common alleles had less increase in LS BMD than those with heterozygous genotypes (TT/TC, $\mathrm{p}=0.018$ ). Participants with homozygous minor alleles showed a trend of much larger increase in LS BMD, but it did not reach statistical significance (CC/TC, $\mathrm{p}=0.059)$. Genotypes at rs865429 locus were associated with percentage change of FN BMD after 12 months of treatment ( $\mathrm{p}=$ 0.030; Figure 3 \& Table 3). Participants with homozygous minor alleles had less increase in FN BMD than those with other genotypes (CC/TC: $\mathrm{p}=0.009$; CC/ TT: $\mathrm{p}=0.014)$. Multiple linear regression indicated that changes of lumbar spine BMD were correlated to rs1234612 polymorphisms of SOST ( $\mathrm{p}=0.004$, $\left.\mathrm{R}^{2}=0.027\right)$, and changes of femoral neck BMD were correlated to rs865429 polymorphisms of SOST ( $\mathrm{p}=$ $\left.0.029, \mathrm{R}^{2}=0.055\right)$. Genotypes at the other SNPs loci were uncorrelated to percentage change of BMD after treatment of alendronate (Supplementary Table 3).

\section{Discussion}

The Wnt signaling pathway is believed to play an important role in bone formation, and Wnt antagonist, scleorostin, modulates this pathway [15]. In this study, the polymorphisms of SOST are found to play equally essential roles on BMD and on bone response to alendronate. The SOST rs1234612 and rs851054 polymorphisms are correlated to baseline BMD at lumbar spine. The SOST rs1234612 and rs865429 
Table 2. Significant correlation between SOST polymorphims and bone turnover markers and bone mineral density at baseline.

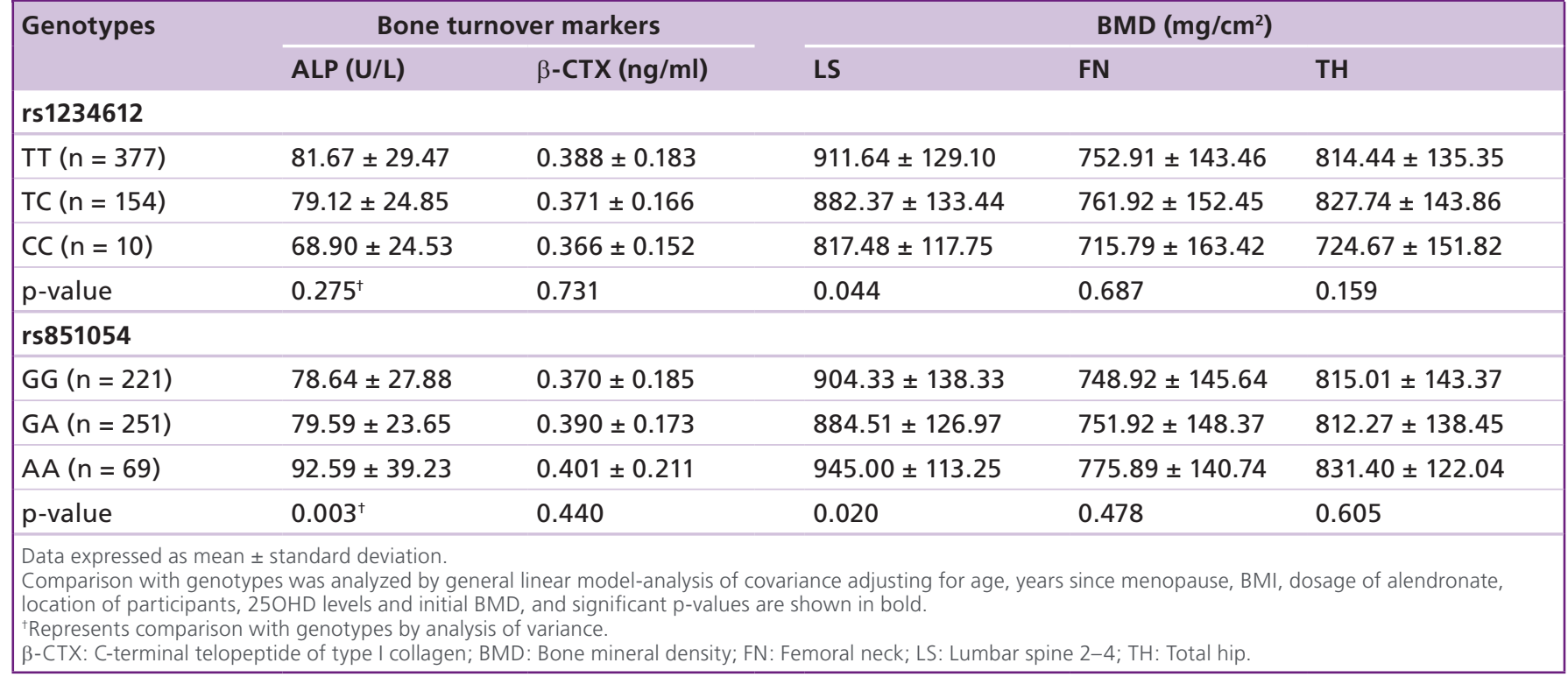

polymorphisms are associated with change of BMD after alendronate treatment. To the best of our knowledge, this is the first study about the correlation of genetic variance in SOST and bone response to BPs. Moreover, we find that the polymorphisms frequencies of SOST in Chinese are similar with those in Japan Tokyo people but different from those in European Caucasians and sub-Saharan Africans (http:// hapmap.ncbi.nlm.nih.gov).

Although alendronate has been demonstrated to be effective in reduction of bone fracture risk in postmenopausal osteoporotic women [27], 25.8\% of whom are inadequate responders to BPs treatment, which indicates that efficacy of BPs is highly heterogeneous and affected by many factors [6]. As we know, alendronate inhibits osteoclasts function through specifically inhibiting crucial enzymes of the intracellular mevalonate pathway, including FDPS and GGPPS. Polymorphisms of FDPS and GGPPS genes have been reported to be correlated with response to alendronate $[11,28]$.

Although alendronate reduces bone fracture incidence through inhibiting functions of osteoclasts, wbone formation is necessary to achieve net gain in bone mass. In a fracture intervention trial, the efficacy of alendronate on reducing nonvertebral fracture risk is greater among women with high baseline levels of N-terminal propeptide of type I collagen, which confirms that osteoblasts activity is also important to keep efficacy of BPs [29]. As we know, Wnt signaling pathway gives a pivotal role in modulating bone formation. Scleorostin, a glycoprotein secreted by osteocytes, offers new therapeutic paradigm for treatment of osteo- porosis as a regulator of Wnt signaling [15] and of bone morphogenetic protein signaling [30]. SOST has been revealed as a susceptibility gene for osteoporosis and osteoporotic fractures in different populations, including in Chinese $[19,20,31,32]$. Moreover, a positive correlation has been reported between incident hip fractures and high sclerostin levels [33,34]. In this study, we also find genetic variance of SOST is correlated to skeletal response to alendronate treatment for the first time. The rs1234612, rs851054 and rs865429 loci locate in the $9.7 \mathrm{~kb}$ region upstream, the 5 promoter region and the first intron of SOST, respectively. They are all revealed as transcription factor binding sites through SNP function prediction web-tools (http://snpinfo. niehs.nih.gov/snpinfo/snpfunc.htm) or (http://fastsnp.ibms.sinica.edu.tw/pages/input_SNPListAnalysis. jsp). These genetic variances may change the expression level of scleorostin, influence bone formation and lead to different baseline BMD and variant bone response to BPs treatment. However, we do not find the consistent correlation between SOST genotypes and changes in bone biomarker, which possibly due to the total alkaline phosphotase is not a sensitive and specific index of bone formation. Serum scleorostin and procollagen type $1 \mathrm{~N}$-terminal propeptide levels should be measured as the meaningful markers in elucidating the mechanism of SOST genotype-related differences in response to treatment. In short, the detail mechanisms of genetic variance of SOST influencing the effects of BPs remain to be explored.

A series of studies investigate the relationship between the SOST genotypes and BMD, and the 


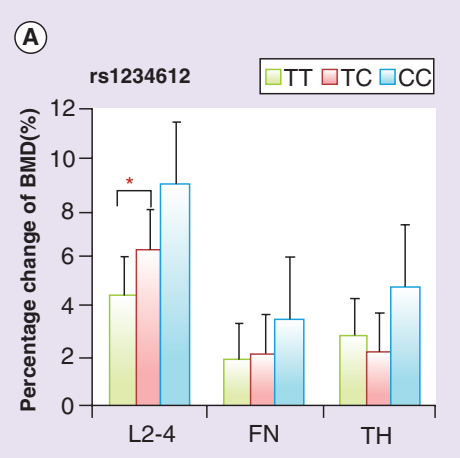

(B)

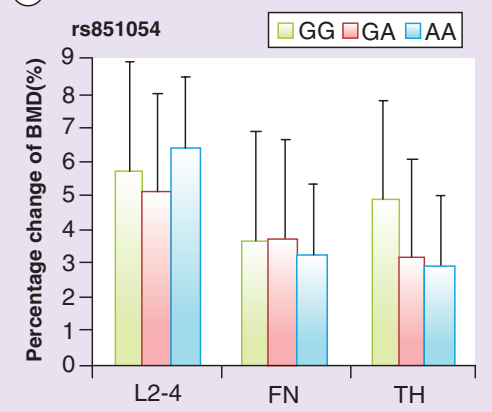

(C)

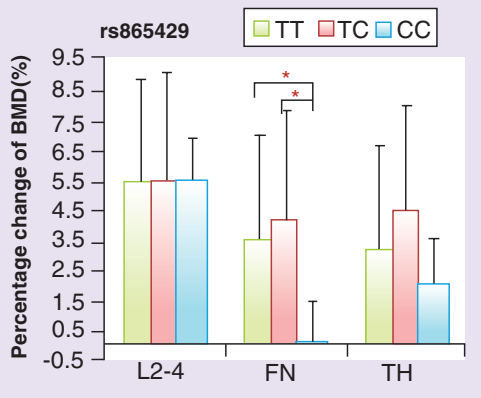

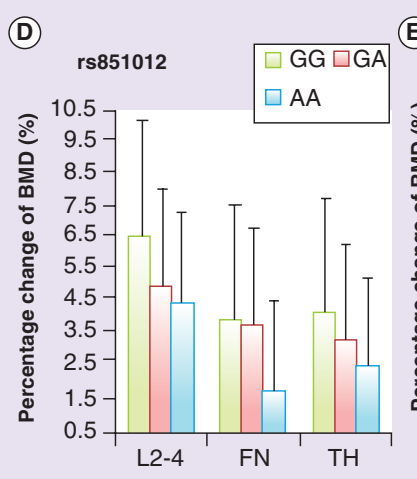
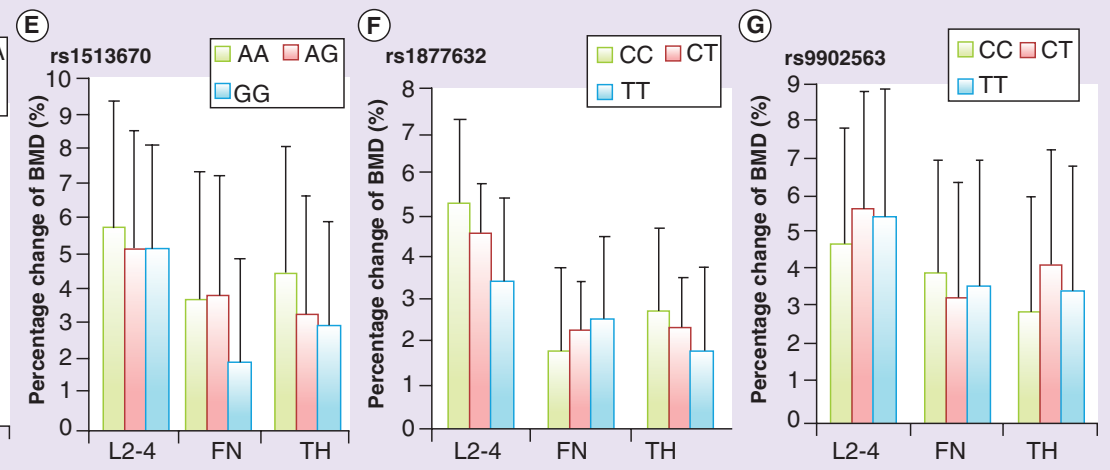

Figure 3. Percentage changes in bone mineral density after 12 months of alendronate treatment in relation to SOST polymorphisms.

FN: Femoral neck; TH: Total hip.

results are inconsistent. We find the SOST rs1234612 polymorphism are significantly associated with baseline lumbar spine BMD, which is not find in healthy postmenopausal Shanghai women [19,35]. rs1513670 polymorphisms of SOST are found to be associated with total hip BMD in healthy Shanghai women [30], but we fail to find this association. We reveal rs851054 polymorphisms of SOST gene significantly correlated to lumbar spine BMD, which are consistent with findings in old American men and postmenopausal Australian women [23,34], but do not conform to results in Korean and Spanish postmenopausal population [36,37]. As osteoporosis is a complex disease influenced by multiple factors, these inconsistent results could mainly stem from the difference in population selection, ethnicity, sample size, age, sexuality, status of menstruation, geographical distribution of participants, scanner types for BMD and so on.

In the same cohort, the LRP5 and FDPS polymorphisms are revealed to be significantly correlated with effects of alendronate treatment $[10,11]$. Sclerosin inhibits Wnt pathway of osteoblasts through competitively binding to LRP5. High bone mass-causing mutations in LRP5 may due to reduced binding and interaction of sclerostin to LRP5 [14]. FDPS is the critical enzyme in the mevalonate pathway and acts as the target of aminobisphosphonates in osteoclasts. Therefore, they are all candidate genes correlated to response to alendronate in our cohort. We deduce the correlation is caused by the effects of genetic variance on bone turnover. It is worthy of study if there are potential genegene interactions or not.

There are several limitations in our study. First, vitamin D levels in this population are very low and only 125 IU vitamin D3 is given daily, which maybe affect BMD gain after treatment. However, it will be beneficial to confirm the pharmacogenomic effects are related to alendronate. Second, the genotype frequency of rs 851062 and rs1513670 of SOST does not conform with HardyWeinberg equilibrium, which could be due to the relative insufficiency of samples. We should investigate the influence of rs851062 and rs1513670 polymorphism of SOST and BMD, bone response to alendronate treatment in a larger sample. As the SNPs frequencies of SOST in Chinese are different from those in European and African, the correlation of SOST polymorphisms and response to BPs should be investigated in the other populations. Third, according to the least significant change value of BMD, 1/3 and 1/4 women in this study are inadequate responders at lumbar spine and total hip to alendronate treatment. The failure of the therapies associated to many factors, such as the hypovitaminosis $\mathrm{D}$, inadequate 
Table 3. Significant correlation between SOST polymorphisms and adjusted percentage changes in bone turnover markers and bone mineral density after 12 months of alendronate treatment.

\begin{tabular}{|c|c|c|c|c|c|}
\hline \multirow[t]{2}{*}{ Genotypes } & \multicolumn{2}{|c|}{ Bone turnover markers (\% per year) } & \multicolumn{3}{|c|}{ BMD (\% per year) } \\
\hline & ALP & $\beta$-СТX & LS & FN & TH \\
\hline \multicolumn{6}{|l|}{ rs1234612 } \\
\hline TT $(n=343)$ & $-29.89 \pm 17.18$ & $-49.68 \pm 37.28$ & $4.50 \pm 4.47$ & $1.88 \pm 1.54$ & $2.84 \pm 0.67$ \\
\hline $\mathrm{TC}(\mathrm{n}=142)$ & $-31.29 \pm 15.39$ & $-46.92 \pm 37.03$ & $6.31 \pm 6.08$ & $2.07 \pm 1.67$ & $2.15 \pm 0.75$ \\
\hline$C C(n=10)$ & $-25.93 \pm 9.84$ & $-56.15 \pm 25.37$ & $8.90 \pm 7.51$ & $3.45 \pm 5.65$ & $4.80 \pm 2.59$ \\
\hline p-value & 0.661 & 0.841 & $0.015^{\dagger}$ & 0.300 & $0.566^{\dagger}$ \\
\hline \multicolumn{6}{|l|}{ rs865429 } \\
\hline $\mathrm{TT}(\mathrm{n}=245)$ & $-26.98 \pm 13.64$ & $-66.44 \pm 26.34$ & $5.38 \pm 3.62$ & $3.47 \pm 3.92$ & $3.15 \pm 3.49$ \\
\hline TC $(n=213)$ & $-26.83 \pm 14.65$ & $-61.66 \pm 42.66$ & $5.42 \pm 5.36$ & $4.12 \pm 3.66$ & $4.36 \pm 3.86$ \\
\hline$C C(n=33)$ & $-21.86 \pm 9.56$ & $-57.14 \pm 38.62$ & $5.41 \pm 2.93$ & $-0.08 \pm 3.16$ & $1.99 \pm 1.53$ \\
\hline p-value & $0.734^{+}$ & $0.756^{\dagger}$ & $0.999^{\dagger}$ & 0.030 & $0.190^{+}$ \\
\hline \multicolumn{6}{|c|}{$\begin{array}{l}\text { Data expressed as mean } \pm \text { standard deviation. } \\
\text { Comparison with genotypes was analyzed by general linear model-analysis of covariance adjusting for age, years since menopause, BMI, } \\
\text { dosage of alendronate, location of participants, } 25(\mathrm{OH}) \mathrm{D} \text { levels and initial BMD, and significant p-values are shown in bold. } \\
\text { 'Represents comparison with genotypes by analysis of variance; } \\
\text { BMD: Bone mineral density; CTX: C-terminal telopeptide of type I collagen; FN: Femoral neck; LS: Lumbar spine 2-4; TH: Total hip. }\end{array}$} \\
\hline
\end{tabular}

adherence, complicated genetic factors. SNPs of $V D R$, $E R \beta, C O L 1 A 1, F D P S, L R P 5$, and so on, are reported to be correlated to the effects of BPs [7]. Therefore, SOST polymorphisms could only forecast parts of inadequate responders. Fourth, the duration of alendronate treatment is rather short, only 1 year. In the end, SOST polymorphisms are valuable makers for alendronate effects and for bone healthy because they are correlated to baseline BMD and percentage changes of BMD after alendronate treatment. As we only evaluate the association of SOST variance and response to alendronate, the influence of SOST polymorphisms and effects of other antiosteoporotic agents need to be investigated further.

There are several strengths in our study. It is the first study to investigate the genotype-treatment interaction between SOST polymorphisms and BPs therapy. This is a prospective, multicenter study in a relatively large sample of postmenopausal women, which could exclude the impacts of many bias factors. We analyze all tag SNP of SOST, which could almost represent the whole genetic variance of SOST. Understanding of the contribution of SOST polymorphisms to effects of alendronate will be helpful to develop appropriate individualized treatment plans on the basis of the genetic characteristics of patients, which will improve the treatment effects and save the medical expenditure.

\section{Conclusion}

The rs1234612 and rs865429 polymorphisms of SOST gene are found for the first time to be correlated to the responsiveness of bone mineral density to alendro- nate treatment. SOST could be an important candidate gene for bone healthy and individualize antiresorptive therapy of osteoporosis.

\section{Future perspective}

Pharmacogenetic studies of osteoporosis are still insufficient. However, it is valuable to predict the effects of antiosteoporotic treatment to ensure maximum efficacy with minimal adverse effects. The SOST gene is a susceptibility gene for osteoporosis. The rs1234612 and rs865429 polymorphisms of SOST gene are found for the first time to be correlated to the responsiveness of bone mineral density to alendronate treatment. SOST could be an important candidate gene for bone healthy and individualize antiresorptive therapy of osteoporosis.

\section{Acknowledgements}

The authors thank Shijiazhuang Quyi Pharmaceutical Co Ltd for supplying alendronate.

Financial \& competing interest disclosure

The authors received financial support from the China National Scientific \& Technological Pillar for Program (2006BAI02B03) and National Science Foundation of China (81100623). The authors have no other relevant affiliations or financial involvement with any organization or entity with a financial interest in or financial conflict with the subject matter or materials discussed in the manuscript apart from those disclosed.

No writing assistance is utilized in the production of this manuscript. 
Ethical conduct of research

The authors have obtained appropriate institutional review board approval or have followed the principles outlined in the Declaration of Helsinki for all human or animal experimental investigations. In addition, for investigations involving human subjects, informed consents have been obtained from the participants involved.

\section{Executive summary}

\section{Background}

- Sclerostin (SOST) is an important antagonist for Wnt pathway in both osteocytes and osteoblast. The polymorphisms of SOST gene are associated with bone mineral density (BMD) in different ethnic populations. Little information is available about whether SOST variants affect the response to alendronate treatment.

\section{Materials \& methods}

- Patients: 639 Chinese postmenopausal women with osteoporosis or osteopenia were enrolled.

- Treatment: all participants randomly received $70 \mathrm{mg} /$ week or $70 \mathrm{mg} / 2$ weeks of alendronate for 1 year. All women were also supplemented with $600 \mathrm{mg}$ calcium and $125 \mathrm{IU}$ vitamin D3 daily.

- Polymorphisms of SOST gene determination: six tag SNPs and one positive SNP were investigated by TaqMan allelic discrimination assay.

- Assessment of response to treatment: BMD and bone turnover biomarkers (C-telopeptide of type I collagen, $\beta$-CTX and ALP) were measured at baseline and during follow-up.

\section{Results}

- rs1234612 and rs851054 polymorphisms are correlated to baseline lumbar spine BMD $(p<0.05)$. After 12 months of treatment, rs1234612 and rs865429 polymorphisms are correlated to BMD changes at lumbar spine $(p<0.05)$ or femoral neck $(p<0.05)$, respectively.

\section{Conclusion}

- The rs1234612 and rs865429 polymorphisms of SOST gene are found for the first time to be correlated to the responsiveness of bone mineral density to alendronate treatment. SOST could be an important candidate gene for bone healthy and individualize antiresorptive therapy of osteoporosis.

\section{References}

Papers of special note have been highlighted as:

- of interest; $\bullet \bullet$ of considerable interest

1 NIH Consensus Development Panel on Osteoporosis Prevention Diagnosis and Therapy. Osteoporosis prevention, diagnosis, and therapy. JAMA 285(6), 785-795 (2001).

2 Moayyeri A, Hsu Y-H, Karasik D et al. Genetic determinants of heel bone properties: genome-wide association metaanalysis and replication in the GEFOS/GENOMOS consortium. Hum. Mol. Genet. 23(11), 3054-3068 (2014).

- Meta-analysis revealing that several genes had genomewide significant associations with heel bone properties. The results confirmed the genetic contribution to osteoporosis.

3 Estrada K, Styrkarsdottir U, Evangelou E et al. Genome-wide meta-analysis identifies 56 bone mineral density loci and reveals 14 loci associated with risk of fracture. Nat. Genet. 44(5), 491-501 (2012).

- Meta-analysis showing that 56 loci associated with bone mineral density (BMD) at genome-wide significant level. Wnt factors are known to be associated with BMD, in which the candidate genes include SOST, CTNNB1, LRP4, LRP5, WLS, WNT4, MEF2C.

4 Styrkarsdottir U, Halldorsson BV, Gretarsdottir S et al. Multiple genetic loci for bone mineral density and fractures. N. Engl. J. Med. 358(22), 2355-2365 (2008).

- Reports on a study about the effect of common sequence variants on BMD and on low-trauma fractures in three populations of European population, which confirmed the genetic contribution to osteoporosis
5 Richards JB, Rivadeneira F, Inouye M et al. Bone mineral density, osteoporosis, and osteoporotic fractures: a genomewide association study. Lancet 371(9623), 1505-1512 (2008).

-. This study identified genome-wide evidence for an association between bone mineral density and two SNPs, which were rs 4355801 , on chromosome 8 , near to the TNFRSF11B gene, and rs3736228, on chromosome 11 in the LRP5 gene. It indicates that Wnt pathway is important to genetic susceptibility to osteoporosis.

6 Cairoli E, Eller-Vainicher C, Ulivieri FM et al. Factors associated with bisphosphonate treatment failure in postmenopausal women with primary osteoporosis. Osteoporos. Int. 25(4), 1401-1410 (2014).

7 Marini F, Brand ML. Pharmacogenetics of osteoporosis: future perspectives. Calcif. Tissue Int. 84(5), 337-347 (2009).

- Review showing that responses to bisphosphonates could relate to genetic background. Pharmacogenetic studies are useful tools to make decisions about bisphosphonate treatment.

8 Creatsa M, Pliatsika P, Kaparos G et al. The effect of vitamin $\mathrm{D}$ receptor BSMI genotype on the response to osteoporosis treatment in postmenopausal women: a pilot study. J. Obstet Gynaecol. Res. 37(10), 1415-1422 (2011).

9 Arko B, Prezelj J, Komel R, Kocijancic A, Marc J. No major effect of estrogen receptor beta gene RSAI polymorphism on bone mineral density and response to alendronate therapy in postmenopausal osteoporosis. J. Steroid Biochem. Mol. Biol. 81(2), 147-152 (2002). 
10 Zhou PR, Liu HJ, Li M et al. LRP5 polymorphisms and response to alendronate treatment in Chinese postmenopausal women with osteoporosis. Pharmacogenomics 15(6), 821-831 (2014).

-. In the same population of Chinese osteoporotic women, the $\mathrm{A} 1330 \mathrm{~V}$ polymorphism of $L R P 5$ was shown to be correlated with lumbar spine BMD changes after alendronate treatment. LRP5 and sclerostin are essential elements of the Wnt pathway, which suggests that SOST could also be a possible candidate gene related to response to antiosteoporotic treatment.

11 Liu Y, Liu H, Li M et al. Association of farnesyl diphosphate synthase polymorphisms and response to alendronate treatment in Chinese postmenopausal women with osteoporosis. Chin. Med. J. (Engl.) 127(4), 662-668 (2014).

12 Kim H, Lee DO, Ku S-Y, Kim SH, Kim JH, Kim JG. The association between polymorphisms in Wnt antagonist genes and bone response to hormone therapy in postmenopausal Korean women. Menopause 19(9), 1008-1014 (2012).

13 Canalis E. Wnt signaling in osteoporosis: mechanisms and novel therapeutic approaches. Nat. Rev. Endocrinol. 9(10), 575-583 (2013).

14 Ke HZ, Richards WG, Li X, Ominsky MS. Sclerostin and Dickkopf-1 as therapeutic targets in bone diseases. Endocr. Rev. 33(5), 747-783 (2012).

- As a major inhibitor of Wnt pathway and a new therapeutic target of osteoporosis, sclerostin exerts key role on bone formation, which is associated with the mechanism of $S O S T$ polymorphisms affecting the response to alendronate treatment.

15 Monroe DG, McGee-Lawrence ME, Oursler MJ, Westendorf JJ. Update on Wnt signaling in bone cell biology and bone disease. Gene 492(1), 1-18 (2012).

16 van Lierop AH, Hamdy NA, van Egmond ME, Bakker E, Dikkers FG, Papapoulos SE. Van Buchem disease: clinical, biochemical, and densitometric features of patients and disease carriers. J. Bone Miner. Res. 28(4), 848-854 (2013).

17 McClung MR, Grauer A, Boonen S et al. Romosozumab in postmenopausal women with low bone mineral density. $N$. Engl. J. Med. 370 (5), 412-420 (2014).

18 McColm J, Hu L, Womack T, Tang CC, Chiang AY. Singleand multiple-dose randomized studies of blosozumab, a monoclonal antibody against sclerostin, in healthy postmenopausal women. J. Bone Miner. Res. 29(4), 935-943 (2014).

19 Zhang H, He JW, Wang C et al. Associations of polymorphisms in the SOST gene and bone mineral density in postmenopausal Chinese women. Osteoporos. Int. 25(12), 2797-2803 (2014).

20 Piters E, de Freitas F, Nielsen TL, Andersen M, Brixen K, Van Hul W. Association study of polymorphisms in the SOST gene region and parameters of bone strength and body composition in both young and elderly men: data from the Odense Androgen Study. Calcif. Tissue Int. 90(1), 30-39 (2012).

21 Serrano AJ, Begona L, Anitua E, Cobos R, Orive G. Systematic review and meta-analysis of the efficacy and safety of alendronate and zoledronate for the treatment of postmenopausal osteoporosis. Gynecol. Endocrinol. 29(12), 1005-1014 (2013).

22 Barrett JC, Fry B, Maller J, Daly MJ. Haploview: analysis and visualization of LD and haplotype maps. Bioinformatics 21(2), 263-265 (2005).

23 Yerges LM, Klei L, Cauley JA et al. High-density association study of 383 candidate genes for volumetric BMD at the femoral neck and lumbar spine among older men. J. Bone Miner. Res. 24(12), 2039-2049 (2009).

-• Shows that the rs851054 polymorphism of SOST was associated with the variation in lumbar spine BMD after adjusting confounding factors, which was similar to our results.

24 Zmuda JM, Yerges-Armstrong LM, Moffett SP et al. Genetic analysis of vertebral trabecular bone density and cross-sectional area in older men. Osteoporos. Int. 22(4), 1079-1090 (2011).

25 Genant HK, Wu CY, van Kuijk C, Nevitt MC. Vertebral fracture assessment using a semiquantitative technique. J. Bone Miner. Res. 8(9), 1137-1148 (1993).

26 Li M, Zhang ZL, Liao EY et al. Effect of low-dose alendronate treatment on bone mineral density and bone turnover markers in Chinese postmenopausal women with osteopenia and osteoporosis. Menopause 20 (1), 72-78 (2013).

27 Bone HG, Hosking D, Devogelaer JP et al. Ten years' experience with alendronate for osteoporosis in postmenopausal women. N. Engl. J. Med. 350(12), 1189-1199 (2004).

28 Choi HJ, Choi JY, Cho SW et al. Genetic polymorphism of geranylgeranyl diphosphate synthase (GGSP1) predicts bone density response to bisphosphonate therapy in Korean women. Yonsei. Med. J. 51(2), 231-238 (2010).

29 Bauer DC, Garnero P, Hochberg MC et al. Pretreatment levels of bone turnover and the antifracture efficacy of alendronate: the fracture intervention trial. J. Bone Miner. Res. 21(2), 292-299 (2006).

30 Liu JM, Zhang MJ, Zhao L et al. Analysis of recently identified osteoporosis susceptibility genes in Han Chinese women. J. Clin. Endocrinol. Metab. 95(9), E112-E120 (2010).

31 Reppe S, Noer A, Grimholt RM et al. Methylation of bone SOST, its mRNA, and serum sclerostin levels correlate strongly with fracture risk in postmenopausal women. J. Bone Miner. Res. 30 (2), 249-256 (2014).

32 Amrein K, Dobnig H, Wagner D et al. Sclerostin in institutionalized elderly women: associations with quantitative bone ultrasound, bone turnover, fractures, and mortality. J. Am. Geriatr. Soc. 62(6), 1023-1029 (2014).

33 Ardawi MS, Rouzi AA, Al-Sibiani SA, Al-Senani NS, Qari MH, Mousa SA. High serum sclerostin predicts the occurrence of osteoporotic fractures in postmenopausal women: the Center of Excellence for Osteoporosis Research Study. J. Bone Miner. Res. 27(12), 2592-2602 (2012).

34 Sims AM, Shephard N, Carter K et al. Genetic analyses in a sample of individuals with high or low BMD shows association with multiple Wnt pathway genes. J. Bone Miner. Res. 23(4), 499-506 (2008). 
- $\quad$ Reports on the association of multiple Wnt pathway genes with BMD in Australian populations. The rs851054 polymorphism of $S O S T$ was associated with high BMD, which is similar to our results.

35 He J, Zhang $\mathrm{H}$, Wang $\mathrm{C}$ et al. Associations of serum sclerostin and polymorphisms in the SOST gene with bone mineral density and markers of bone metabolism in postmenopausal Chinese women. J. Clin. Endocrinol. Metab. 99(4), E665-E673 (2014).
36

Lee DO, Kim H, Ku SY, Kim SH, Kim JG. Association between polymorphisms in sclerostin, dickkopfs and secreted frizzled-related protein genes and bone mineral density in postmenopausal Korean women. Gynecol. Obstet. Invest. 77(3), 186-193 (2014).

37 Valero C, Zarrabeitia MT, Hernandez JL et al. Relationship of sclerostin and secreted frizzled protein polymorphisms with bone mineral density: an association study with replication in postmenopausal women. Menopause 18(7), 802-807 (2011). 